PROCEEDINGS OF THE

AMERICAN MATHEMATICAL SOCIETY

Volume 125, Number 12, December 1997, Pages 3741-3746

S 0002-9939(97)04013-6

\title{
A NON-PSEUDOCOMPACT PRODUCT OF COUNTABLY COMPACT SPACES VIA SEQ
}

\author{
W. F. LINDGREN AND A. A. SZYMANSKI
}

(Communicated by Franklin D. Tall)

\begin{abstract}
We show that under Martin's axiom there are $2^{2^{\omega}}$ spaces which are countably compact extremally disconnected homogeneous such that the product of any two them is not pseudocompact. The spaces are modeled on the space $\operatorname{Seq}(\xi)$.
\end{abstract}

Since the discovery in 1981 by Frolik-Comfort that regular (i.e., $T_{1}$ and regular) homogeneous extremally disconnected spaces cannot exist among compact spaces, there has been some effort to relax the compactness property. W.Comfort and J.van Mill [CM] showed in 1987 that regular extremally disconnected homogeneous spaces can exist among countably compact spaces. Their method for constructing such a space was as follows: start with an arbitrary regular homogeneous extremally disconnected space, take its Čech-Stone compactification and, within it, take orbit of a point of the original space. What you get is what you want provided that the original space has a point accessible by a discrete countable subset. There are not many available regular homogeneous extremally disconnected spaces satisfying the accessibility condition. Basically, all known examples have been instances of one such space, $\operatorname{Seq}(\xi)$, where $\xi$ is a free ultrafilter on $\omega$. Therefore any attempt of constructing a regular homogeneous extremally disconnected countably compact space had to appeal ultimately to the space $\operatorname{Seq}(\xi)$. Besides Comfort and van Mill's original example, there was one by A.Kato from 1992 [Ka].

The lack of a variety of examples of regular homogeneous extremally disconnected countably compact spaces is not accidental. We prove that among (locally) separable such spaces the only ones possible are those modeled on the space $\mathbf{S e q}(\xi)$. More precisely, we show that any regular extremally disconnected countably compact separable homogeneous space contains a dense subspace homeomorphic to a Seq $(\xi)$-like space (cf. Theorem 1). We also show that, under Martin's axiom, there exist two regular spaces which are extremally disconnected countably compact separable homogeneous and such that their product is not pseudocompact. This provides an answer to one of Comfort-van Mill's questions [CM, Question $5.2]$.

Received by the editors July 14, 1994.

1991 Mathematics Subject Classification. Primary 54D80, 54B10, 54G05.

Key words and phrases. Countably compact, extremally disconnected, homogeneous.

(C) 1997 American Mathematical Society 


\section{The SPaCe $\operatorname{Seq}(\xi)$ and its modifications}

Let Seq denote the set of all finite sequences of natural numbers, $\omega$, including the empty sequence $\emptyset$. If $\xi$ is an ultrafilter on $\omega$, then $\operatorname{Seq}(\xi)$ denotes the topological space with the underlying set Seq and topology defined as follows:

$U$ is open provided that for every $s \in U,\{n \in \omega: s \frown n \in U\} \in \xi$.

Here $s^{\frown} n$ denotes the concatenation of the sequence $s$ and the sequence $\{n\}$. More generally, if $s$ and $t$ are two finite sequences, then $s \neg t$ denotes the concatenation of the sequences $s$ and $t$; i.e., the sequence $s$ followed by the sequence $t$. It is known that the space $\mathbf{S e q}(\xi)$ is regular, homogeneous and extremally disconnected (cf. [DGS] and [Ka]).

To facilitate our discussion we distinguish two special subspaces of the space $\operatorname{Seq}(\xi)$. For each natural number $n, L_{n}$ denotes the set of all sequences of length exactly $n$ and $T_{n}$ the set of all sequences of length at most $n$. Treated as subspaces of $\operatorname{Seq}(\xi), T_{n}$ is closed and nowhere dense, and $L_{n}$ is relatively discrete and dense in $T_{n}$. If $t \in \mathbf{S e q}(\xi)$, then $C(t)=\{s \in \mathbf{S e q}(\xi): t \subseteq s\}$ - the cone above $t$.

A topological space $X=(\mathbf{S e q}, \tau)$ is said to be $\operatorname{Seq}(\xi)$-like space if $X$ has the following properties:

(i) $X$ is regular and extremally disconnected;

(ii) if $U$ is in $\tau$, then for every $s \in U,\{n \in \omega: s \frown n \in U\} \in \xi$;

(iii) for each $t \in$ Seq, $C(t)$ is a closed-open subset of $X$.

One can easily see that any topology on the set Seq that makes it a $\mathbf{S e q}(\xi)$-like space is a coarsening of the topology of the space $\mathbf{S e q}(\xi)$. The $\mathbf{S e q}(\xi)$-like spaces are also related to $S_{\omega}$-spaces of Comfort and Garcia-Ferreira (cf. [CG]).

Lemma 1. Let $X=(\boldsymbol{S e q}, \tau)$ be a $\boldsymbol{S e q}(\xi)$-like space. Then $X$ is homeomorphic to the space $\boldsymbol{S e q}(\xi)$ if and only if every nowhere dense subspace of the space $X$ has an isolated point, i.e., it is scattered.

Proof. Suppose $E$ is a nowhere dense subset of $\operatorname{Seq}(\xi)$. We will show that the set of isolated points of the closure of $E$ is dense in the closure of $E$.

Let $U$ be a clopen subset of Seq $(\xi)$ intersecting $c l E$. Then $U \cap c l E$ is not open and so there exists $s \in U \cap c l E$ such that $\left\{n: s^{\frown} n \in U \cap c l E\right\} \notin \xi$. Set $A=\{n$ : $\left.s^{\frown} n \notin U \cap c l E\right\}$. Then $A \in \xi$. For every $n \in A$, let $V_{n}$ be an open set containing $s \frown n$ and disjoint from $U \cap c l E$. Then $V=\{s\} \cup\left\{V_{n}: n \in A\right\}$ is an open neighborhood of $s$ and $V \cap U \cap c l E=\{s\}$. This shows that $s$ is an isolated point of the set $U \cap c l E$, thus $s$ is an isolated point of $c l E$.

If every nowhere dense subset of the space $X$ is scattered, then the identity function $i$ on Seq is an irreducible function from the space $\operatorname{Seq}(\xi)$ onto $X$. Since $X$ is extremally disconnected, $i$ is a homeomorphism.

Theorem 1. Let $X$ be a regular separable extremally disconnected homogeneous space containing a non-closed countable discrete subset. Then the space $X$ contains a dense subspace which is homeomorphic to a $\mathbf{S e q}(\xi)$-like space.

Proof. Take any countable discrete subset $K=\left\{k_{n}: n \in \omega\right\}$ of $X$ with $p$ being its accumulation point. For the sake of some notational clarity, let us identify $K$ with the set $\left\{s: s \in{ }^{1} \omega\right\}$ where $p$ is identified with $\emptyset$. Let $\xi$ be the filter on $\omega$ such that for each open subset $U$ of $X$ containing $\emptyset,\left\{n \in \omega: k_{n} \in U\right\} \in \xi$ (i.e., $\emptyset=\xi-\lim K)$. Since $X$ is regular and extremally disconnected, $\xi$ is actually an ultrafilter on $\omega$. Let $D=\left\{d_{n}: n \in \omega\right\}$ be a dense subset of $X$ with $d_{0}=\emptyset$. There 
exists a family $\left\{U_{s}: s \in{ }^{1} \omega\right\}$ of pairwise disjoint closed-open subsets of $X$ such that $s \in U_{s}$ for each $s \in{ }^{1} \omega$ and $\bigcup\left\{U_{s}: s \in{ }^{1} \omega\right\} \supseteq D-c l K$. Since $X$ is homogeneous, for each $s \in{ }^{1} \omega$ there exists a homeomorphism $h_{s}$ of $X$ onto $X$ such that $h_{s}(\emptyset)=s$. Without loss of generality, we may assume that $h_{s}(K) \subseteq U_{s}$ and that $h_{s}\left(k_{0}\right)$ is the element of $U_{s} \cap D$ with the least index. We denote $h_{s}\left(k_{n}\right)$ by $s^{\frown} n$ for each $n \in \omega$ and $s \in{ }^{1} \omega$.

Suppose $n \in \omega, n \geq 1$, and for each $m \leq n$ and $s \in{ }^{m} \omega$ we have defined a closed-open subset $U_{s}$ of $X$ and a point of $U_{s}$, denoted by $s$, such that the following conditions hold:

(a) if $m+1 \leq n$ and $s \in{ }^{m} \omega$, then the family $\left\{U_{s \frown k}: k \in \omega\right\}$ is a pairwise disjoint family of closed-open subsets of $U_{s}$ such that $\bigcup\left\{U_{s \frown k}: k \in \omega\right\} \supseteq$ $(D-\operatorname{cl}\{s \frown k: k \in \omega\}) \cap U_{s}$;

(b) $s \frown 0$ is the element of $U_{s} \cap D$ with the least index;

(c) $s=\xi-\lim \{s \frown k: k \in \omega\}$.

Now fix $s \in{ }^{n} \omega$. There exists a homeomorphism $h_{s}$ of $X$ onto $X$ such that $h_{s}(p)=s$. Without loss of generality, we may assume that $h_{s}(K) \subseteq U_{s}$ and that $h_{s}\left(k_{0}\right)$ is the element of $U_{s} \cap D$ with the least index. We denote $h_{s}\left(k_{m}\right)$ by $s \frown m$ for each $m \in \omega$. There exists a family $\left\{U_{s-m}: m \in \omega\right\}$ of closed-open subsets of $U_{s}$ such that $\bigcup\left\{U_{s \frown m}: m \in \omega\right\} \supseteq(D-c l\{s \frown m: m \in \omega\}) \cap U_{s}$. Thus for each $m \leq n+1$ and $s \in{ }^{m} \omega$ we have defined a closed-open subset $U_{s}$ of $X$ and a point of $U_{s}$, denoted by $s$, such that the conditions (a), (b) and (c) are satisfied. The induction is finished.

Now we shall show that the set Seq considered as a subset of the space $X$ with the subspace topology $\tau$ is a dense $\operatorname{Seq}(\xi)$-like space.

To this end, it follows from (a) and (b) that the closure of Seq contains $D$ and thus (Seq, $\tau)$ is dense in $X$ and extremally disconnected. It follows from the construction that $C(t)=U_{t} \cap$ Seq for each $t \in$ Seq. Hence $C(t)$ is closed-open for each $t \in$ Seq. Taking into consideration (c), (Seq, $\tau$ ) satisfies all conditions (i)-(iii) to be a $\operatorname{Seq}(\xi)$-like space.

\section{THE SPACE $H(\xi)$}

If $X$ is a completely regular space, then $X^{*}$ denotes the remainder of the CechStone compactification, $\beta X$, of the space $X$. In particular, Seq* $(\xi)=\beta \mathbf{S e q}(\xi)-$ $\operatorname{Seq}(\xi)$.

For a free ultrafilter $\xi$ on $\omega$ we set: $H(\xi)=\{h(\emptyset): h \in A u t(\beta \operatorname{Seq}(\xi))\}$, where $\operatorname{Aut}(\beta \mathbf{S e q}(\xi))$ denotes the group of autohomeomorphisms of the space $\beta \mathbf{S e q}(\xi)$. In other words, the space $H(\xi)$ is the orbit of the point $\emptyset$ in the space $\beta \mathbf{S e q}(\xi)$. This space has been distinguished by Comfort and van Mill, $[\mathrm{CM}]$, in connection with their work on the existence of homogeneous extremally disconnected spaces. It is known that every space $H(\xi)$ is extremally disconnected countably compact separable and homogeneous (cf. [Ka] and [LS]). By specifying ultrafilters $\xi$, one can get more properties of the spaces $H(\xi)$. In particular, A.Kato [Ka] showed that if $\xi_{1}$ and $\xi_{2}$ are weak P-points of different type, then the product $H\left(\xi_{1}\right) \times H\left(\xi_{2}\right)$ is not countably compact. It turns out it can be even made non-pseudocompact provided the ultrafilters are selective (see Theorem 2, below). This constitutes an answer to a question of Comfort and van Mill.

Let us recall some definitions pertaining to ultrafilters on $\omega$. We say that ultrafilters $\xi_{1}$ and $\xi_{2}$ are of the same type if there exists a permutation $f$ of $\omega$ such 
that $\beta f\left(\xi_{1}\right)=\xi_{2}$. The Rudin-Frolik order $\sqsubset$ on $\omega^{*}$ is defined in the following way: for $\xi, \zeta \in \omega^{*}, \xi \sqsubset \zeta$ provided that there is an embedding $h: \beta \omega \rightarrow \omega^{*}$ such that $h(\xi)=\zeta$. We say that $\xi$ is a weak P-point of $\beta \omega$ if $\xi \in \omega^{*}$ and $\xi \notin c l D$ for any countable subset of $\omega^{*}$ not containing $\xi$. We say that an ultrafilter $\xi$ on $\omega$ is selective if for every function $f \in \omega_{\omega}$ there is $A \in \xi$ such that either $f$ is one-to-one on $A$ or $f$ is constant on $A$. Any selective ultrafilter is a weak P-point and it is known selective ultrafilters exist in the number $2^{2^{\omega}}$ assuming Martin's axiom (cf. [Ku]).

For any $s \in$ Seq, let the set $\{s \frown n: n \in \omega\}$ be identified with $\omega$ via the map $f\left(s^{\frown} n\right)=n$. Then the closure of $\left\{s^{\frown} n: n \in \omega\right\}$ in $\beta \mathbf{S e q}(\xi)$ can be identified with $\beta \omega$ and the point $s$ with the ultrafilter $\xi$. We are going to exploit this identification in the sequel, usually without explicit mention.

The lemma below is known; it follows from a result by M.E.Rudin [R] (cf. also [CN, Theorem 16.16]).

Lemma 2. Let $\xi$ and $\zeta$ be weak P-points of $\omega^{*}$ such that $\xi \sqsubset p$ and $\zeta \sqsubset p$ for some $p \in \omega^{*}$. Then $\xi$ and $\zeta$ are of the same type.

Lemma 3. Let $X$ and $Y$ be completely regular spaces without isolated points. Suppose that there exist families $\left\{U_{n}: n \in \omega\right\}$ and $\left\{V_{n}: n \in \omega\right\}$ of non-empty open subsets of $X$ and $Y$, respectively, such that:

(i) $c l U_{n} \cap c l U_{m}=\emptyset=c l V_{n} \cap c l V_{m}$ for each $n, m \in \omega, n \neq m$,

(ii) if $p \in c l \bigcup\left\{U_{n}: n \in \omega\right\}-\bigcup\left\{U_{n}: n \in \omega\right\}$ and $q \in \operatorname{cl} \bigcup\left\{V_{n}: n \in \omega\right\}-$ $\bigcup\left\{V_{n}: n \in \omega\right\}$, then there are open neighborhoods $U$ of $p$ and $V$ of $q$ such that $\left\{n: U \cap U_{n} \neq \emptyset\right\} \cap\left\{n: V \cap V_{n} \neq \emptyset\right\}=\emptyset$.

Then the product $X \times Y$ is a non-pseudocompact space.

Proof. It can be easily shown that if $\left\{U_{n}: n \in \omega\right\}$ and $\left\{V_{n}: n \in \omega\right\}$ are families satisfying (i) and (ii), then the family $\left\{U_{n} \times V_{n}: n \in \omega\right\}$ is locally finite in the space $X \times Y$. Thus the space $X \times Y$ is not pseudocompact.

Theorem 2. If $\xi_{1}$ and $\xi_{2}$ are selective ultrafilters of different type, then the product $H\left(\xi_{1}\right) \times H\left(\xi_{2}\right)$ is not pseudocompact.

Proof. Let $C^{i}(s)$ denote the closure of the cone $C(s)$ in the space $H\left(\xi_{i}\right)$ for $i=1,2$. Clearly, $C^{i}(s)$ is a closed-open subset of the space $H\left(\xi_{i}\right)$ for each $i \in\{1,2\}$ and $s \in$ Seq. We shall show that the family $\left\{C^{1}(s) \times C^{2}(s): s \in L_{1}\right\}$ is a locally finite family in $H\left(\xi_{1}\right) \times H\left(\xi_{2}\right)$ by showing that the families $\left\{C^{1}(s): s \in L_{1}\right\}$ and $\left\{C^{2}(s): s \in L_{1}\right\}$ satisfy the conditions (i) and (ii) of Lemma 3.

To facilitate our further discussion we introduce a function $\pi$ : Seq $\rightarrow L_{1}$ given by the rule $\pi(t)=t\left\lceil 1\right.$. We set $\pi^{i}: \beta \operatorname{Seq}\left(\xi_{i}\right) \rightarrow \beta L_{1}, i \in\{1,2\}$, to be the continuous extension of the function $\pi$.

Let $(p, q) \in H\left(\xi_{1}\right) \times H\left(\xi_{2}\right)$ and assume that $p \notin \bigcup\left\{C^{1}(s): s \in L_{1}\right\}=C^{1}$ and that $q \notin \bigcup\left\{C^{2}(s): s \in L_{1}\right\}=C^{2}$. Thus $\pi^{1}(p) \in L_{1}^{*}$ and $\pi^{2}(q) \in L_{1}^{*}$

Claim 1. The ultrafilters $\pi^{1}(p)$ and $\xi_{1}$ are of the same type or $\xi_{1} \sqsubset \pi^{1}(p)$.

Proof of the Claim. There exists an $h \in \operatorname{Aut}\left(\beta \mathbf{S e q}\left(\xi_{1}\right)\right)$ such that $h(\emptyset)=p$. We must consider three cases:

(a) There exists a subset $A$ of $L_{1}$ such that $A \in \xi_{1}$ and $h(A) \subseteq C^{1}$. Since $\xi_{1}$ is selective, the function $\pi^{1} \circ h$ must be one-to-one on some $B \in \xi_{1}$ which is a subset of $A$. Hence $\pi^{1} \circ h\left\lceil B\right.$ witnesses that $\pi^{1}(p)$ and $\xi_{1}$ are of the same type.

(b) There exists a subset $A$ of $L_{1}$ such that $A \in \xi_{1}, h(A) \subseteq\left(C^{1}\right)^{*}$ and the function $\pi^{1} \circ h$ is not constant on any $B \in \xi_{1}$. Since $\xi_{1}$ is selective, the function 
$\pi^{1} \circ h$ must be one-to-one on some $B \in \xi_{1}$ which is a subset of $A$. Hence $\pi^{1} \circ h\lceil B$ witnesses that $\xi_{1} \sqsubset \pi^{1}(p)$.

(c) There exists a subset $A$ of $L_{1}$ such that $A \in \xi_{1}, h(A) \subseteq\left(C^{1}\right)^{*}$ and the function $\pi^{1} \circ h$ is constant on $A$. Since $p \in \operatorname{clh}(A), \pi^{1}(p)=\pi^{1}(h(a))$ for all $a \in A$. Since the set $N=\left\{x \in H\left(\xi_{1}\right): \pi^{1}(x)=\pi^{1}(p)\right\}$ is nowhere dense, there exists $s \in$ Seq such that $\pi^{1}(h(s))=\pi^{1}(p)$ and $\left\{n \in \omega: h\left(s^{\frown} n\right) \notin N\right\} \in \xi$. Therefore either (a) or (b) applies to $s$. Hence either $\pi^{1}(h(s))$ and $\xi_{1}$ are of the same type or $\xi_{1} \sqsubset \pi^{1}(h(s))$. Since $\pi^{1}(h(s))=\pi^{1}(p), \pi^{1}(p)$ and $\xi_{1}$ are of the same type or $\xi_{1} \sqsubset \pi^{1}(p)$.

A similar argument establishes the following claim.

Claim 2. The ultrafilters $\pi^{2}(q)$ and $\xi_{2}$ are of the same type or $\xi_{2} \sqsubset \pi^{2}(q)$.

It follows that the ultrafilters $\pi^{1}(p)$ and $\pi^{2}(q)$ are different. Indeed, if they were not, then one of the following would hold:

(i) The ultrafilters $\pi^{1}(p)$ and $\xi_{1}$ are of the same type and the ultrafilters $\pi^{2}(q)$ and $\xi_{2}$ are of the same type; hence the ultrafilters $\xi_{1}$ and $\xi_{2}$ would be of the same type.

(ii) The ultrafilters $\pi^{1}(p)$ and $\xi_{1}$ are of the same type and $\xi_{2} \sqsubset \pi^{2}(q)$; hence there would be $\xi_{1} \sqsupset \xi_{2}$.

(iii) The ultrafilters $\pi^{2}(q)$ and $\xi_{2}$ are of the same type and $\xi_{1} \sqsubset \pi^{1}(p)$; hence there would be $\xi_{1} \sqsubset \xi_{2}$.

(iv) $\xi_{1} \sqsubset \pi^{1}(p)$ and $\xi_{2} \sqsubset \pi^{2}(q)$; hence the ultrafilters $\xi_{1}$ and $\xi_{2}$ would be of the same type because of Lemma 2 .

There exist disjoint subsets $A$ and $B$ of $L_{1}$ such that $A \in \pi^{1}(p)$ and $B \in \pi^{2}(q)$. Hence $U=\left(\pi^{1}\right)^{-1}(\operatorname{cl} A)$ and $V=\left(\pi^{2}\right)^{-1}(\operatorname{cl} B)$ are closed-open sets containing $p$ and $q$, respectively. Clearly, $U \times V$ is disjoint from $\bigcup\left\{C^{1}(s) \times C^{2}(s): s \in L_{1}\right\}$.

Theorem 3. Assuming Martin's axiom, there exists a family of $2^{2^{\omega}}$ many regular extremally disconnected separable countably compact homogeneous spaces of cardinality continuum such that the product of any two of them is not pseudocompact.

Remark 1. The spaces $H(\xi)$ constructed by means of weak, and hence selective, ultrafilters are of cardinality continuum. If one wants to produce examples of higher cardinalities that yield non-pseudocompact product, it is enough to apply Kato's trick [Ka].

Remark 2. The existence of (even two) spaces as in Theorem 3, separable or not, remains open in ZFC.

\section{ACKNOWLEDGMENT}

We want to thank the referee for some comments that improved readability of the paper.

\section{REFERENCES}

[CG] W.W.Comfort and S. Garcia-Ferreira, Resolvability: a selective survey and some new results, Topology and its Applications, 74 (1996), 149-167. CMP 97:06

[CM] W.W.Comfort and J.van Mill, A homogeneous extremally disconnected countably compact space, Topology and its Applications, 25(1987), 65-73. MR 88a:54059

[CN] W.W.Comfort and S.Negrepontis, The Theory of Ultrafilters, Springer-Verlag, New York 1974. MR 53:135 
[DGS] A.Dow, A.Gubbi and A.Szymanski, Rigid Stone Spaces within ZFC, Proceedings of the American Mathematical Society 102(1988), 745-748. MR 89d:54028

[Ka] A.Kato, A new construction of extremally disconnected topologies, Topology and its Applications, 58 (1994), 1-16. MR 95i:54042

[Ku] K.Kunen, Set Theory. An introduction to independence proofs, North Holland, Amsterdam 1980. MR 82f:03001

[LS] W.Lindgren and A.Szymanski, On some homogeneous extremally disconnected spaces, Annals of the New York Academy of Sciences, vol. 767(1995), 108-115.

[R] M.E.Rudin, Partial orders on the types of $\beta \mathbf{N}$, Transactions of the American Mathematical Society 155(1971), 353-362. MR 42:8459

Department of Mathematics, Slippery Rock University, Slippery Rock, Pennsylvania 16057

E-mail address: William.Lindgren@sru.edu

E-mail address: Andrzej.Szymanski@sru.edu 